\title{
ON THE AVERAGE OF A CERTAIN WIENER FUNCTIONAL AND A RELATED LIMIT THEOREM IN CALCULUS OF PROBABILITY
}

\author{
BY \\ M. KAC
}

1. Introduction. Recently Cameron and Martin( $\left.{ }^{1}\right)$ have published a series of papers in which they have evaluated averages of various nonlinear functionals over the Wiener space. Their method depends on an interesting transformation theory which they have developed for this purpose. But their method proved inapplicable to the evaluation of the average of the functional

$$
\exp \left\{-z \int_{0}^{1}|x(t)| d t\right\} \text {. }
$$

In this paper we shall obtain an expression in closed form which gives the average of (1) for $z>0$. In fact, we shall prove that for $z>0$ the average of (1) is given by the formula

$$
\sum_{j=1}^{\infty} \kappa_{j} \exp \left\{-\delta_{j} z^{2 / 3}\right\}
$$

where $\delta_{1}, \delta_{2}, \delta_{3}, \ldots$ are the positive roots of the derivative of

$$
P(y)=\frac{(2 y)^{1 / 2}}{3}\left\{J_{-1 / 3}\left(\frac{(2 y)^{3 / 2}}{3}\right)+J_{1 / 3}\left(\frac{(2 y)^{3 / 2}}{3}\right)\right\}
$$

and

$$
\kappa_{j}=\left(1+3 \int_{0}^{\delta_{j}} P(y) d y\right) / 3 \delta_{j} P\left(\delta_{j}\right) .
$$

We are unable to extend this result to other values of $z$.

The interest of the result is strengthened by the fact that it is essentially equivalent to the following limit theorem:

If $X_{1}, X_{2}, X_{3}, \cdots$ is a sequence of identically distributed, independent random variables and if $\left({ }^{2}\right)$

Presented to the Society, September 17, 1945; received by the editors August 14, 1945.

(1) See for instance Evaluation of various Wiener integrals by use of certain Sturm-Liouville differential equations, Bull. Amer. Math. Soc. vol. 51 (1945) pp. 73-90, where further references are given.

(2) Whenever convenient we follow the physicists in denoting by $\bar{R}$ the average (mathematical expectation) of $R$. We also use the customary symbol $E(R)$. 


$$
\bar{X}_{j}=0, \quad \overline{X_{j}^{2}}=1,
$$

then the distribution function of

$$
\frac{\left|X_{1}\right|+\left|X_{1}+X_{2}\right|+\cdots+\left|X_{1}+\cdots+X_{n}\right|}{n^{8 / 2}}
$$

approaches, as $n \rightarrow \infty$, a limiting distribution function $\sigma(\alpha)$ whose Laplace transform

is given by formula (2).

$$
\int_{0}^{\infty} e^{-z \alpha} d \sigma(\alpha)
$$

We have not succeeded in finding a reasonably simple formula for $\sigma(\alpha)$. It may be worthwhile to sketch here briefly the procedure followed in this paper.

Since

$$
\int_{0}^{1}|x(t)| d t
$$

is known to be a measurable functional over the Wiener space, it possesses a distribution function $\sigma(\alpha)$ defined as the Wiener measure of the set of those functions $x(t)$ for which

$$
\int_{0}^{1}|x(t)| d t<\alpha
$$

It then follows by a simple argument that the limit theorem stated above holds if the $X$ 's are assumed to be normally distributed. We next show without the assumption of normality that the distribution function of (5) approaches $\sigma(\alpha)$ at each continuity point of $\sigma(\alpha)$. Hence for $X^{\prime}$ 's distributed arbitrarily (apart from satisfying the conditions of the theorem) we have

$$
\begin{aligned}
\lim _{n \rightarrow \infty} E\left(\operatorname { e x p } \left\{-\frac{z}{n^{8 / 2}}\left(\left|X_{1}\right|\right.\right.\right. & \left.\left.+\left|X_{1}+X_{2}\right|+\cdots+\left|X_{1}+\cdots+X_{n}\right|\right\}\right) \\
& =\int_{0}^{\infty} e^{-z \alpha} d \sigma(\alpha) .
\end{aligned}
$$

We then introduce the crucial idea which consists in assuming

$$
\text { Prob. }\left\{X_{j}<\alpha\right\}=2^{-1 / 2} \int_{-\infty}^{\alpha} e^{-2^{1 / 2}|u|} d n \quad(j=1,2, \cdots)
$$

in which case the average

(8) $A_{n}(z)=E\left(\exp \left\{-\frac{z}{n^{8 / 2}}\left(\left|X_{1}\right|+\left|X_{1}+X_{2}\right|+\cdots+\left|X_{1}+\cdots+X_{n}\right|\right)\right\}\right)$ 
can be explicitely calculated in terms of Bessel functions. The passage to the limit is then carried out directly using some rather delicate results from the theory of Bessel functions of high order. tion.

The explicit calculation of (8) depends on solving a certain integral equa-

2. Preliminary considerations. Since the elements of the Wiener space are continuous we have

$$
\int_{0}^{1}|x(t)| d t=\lim _{n \rightarrow \infty} \frac{1}{n} \sum_{k=1}^{n}\left|x\left(\frac{k}{n}\right)\right|
$$

and hence the distribution function of

$$
\frac{1}{n} \sum_{k=1}^{n}\left|x\left(\frac{k}{n}\right)\right|
$$

approaches the distribution function $\sigma(\alpha)$ of

$$
\int_{0}^{1}|x(t)| d t
$$

Since by the definition of Wiener measure the quantities

$$
x\left(\frac{1}{n}\right), x\left(\frac{2}{n}\right)-x\left(\frac{1}{n}\right), \cdots, x(1)-x\left(\frac{n-1}{n}\right)
$$

are independent Gaussian variables each having mean 0 and standard deviation $1 / n^{1 / 2}$, and since

$$
x\left(\frac{k}{n}\right)=x\left(\frac{1}{n}\right)+\left(x\left(\frac{2}{n}\right)-x\left(\frac{1}{n}\right)\right)+\cdots+\left(x\left(\frac{k}{n}\right)-x\left(\frac{k-1}{n}\right)\right)
$$

it follows immediately that if

$$
G_{1}, G_{2}, G_{3}, \cdots
$$

are independent Gaussian variables each having mean 0 and standard deviation 1 , then the distribution function of

$$
\frac{\left|G_{1}\right|+\left|G_{1}+G_{2}\right|+\cdots+\left|G_{1}+G_{2}+\cdots+G_{n}\right|}{n^{3 / 2}}
$$

is the same as that of

$$
\frac{1}{n} \sum_{k=1}^{n}\left|x\left(\frac{k}{n}\right)\right|
$$

Thus the distribution function of (9) approaches $\sigma(\alpha)$.

We now show that if $X_{1}, X_{2}, X_{3}, \cdots$ are independent identically distributed random variables such that 


$$
\bar{X}_{j}=0, \quad \overline{X_{j}^{2}}=1,
$$

then the distribution function of

$$
\frac{\left|X_{1}\right|+\left|X_{1}+X_{2}\right|+\cdots+\left|X_{1}+\cdots+X_{n}\right|}{n^{3 / 2}}
$$

approaches $\sigma(\alpha)$ at each point of continuity of the latter. My original proof of this statement was based on rather tedious moment considerations which required the assumption that all moments of $X_{j}$ are finite.

After the writing of this paper was completed Dr. Erdös and myself have found a way to circumvent the use of the moments. Our method proved to be applicable to several other limit theorems and we have recently submitted our results for publication in the Bulletin of the American Mathematical Society in a note entitled On certain limit theorems of the theory of probability. In this note we show, among other things, that if

$$
Q_{n}(\alpha)=\text { Prob. }\left\{\frac{\left|X_{1}\right|+\left|X_{1}+X_{2}\right|+\cdots+\left|X_{1}+\cdots+X_{n}\right|}{n^{8 / 2}}<\alpha\right\}
$$

then for every integer $k \geqq 1$ and every $\epsilon>0$ one has

$$
\begin{aligned}
& \text { Prob. }\left\{\frac{\left|H_{1}\right|+\cdots+\left|H_{k}\right|}{k^{3 / 2}}<\alpha-\epsilon\right\}-\frac{C}{\epsilon k^{1 / 2}} \leqq \liminf _{n \rightarrow \infty} Q_{n}(\alpha) \\
& \leqq \limsup _{n \rightarrow \infty} Q_{n}(\alpha) \leqq \text { Prob. }\left\{\frac{\left|H_{1}\right|+\cdots+\left|H_{k}\right|}{k^{3 / 2}}<\alpha+\epsilon\right\}+\frac{C}{\epsilon k^{1 / 2}},
\end{aligned}
$$

where $H_{j}=G_{1}+G_{2}+\cdots+G_{j}$ and $C$ is a certain constant.

If in (11) we let $k$ approach $\infty$, keeping $\epsilon$ fixed, we obtain

$$
\sigma(\alpha-\epsilon) \leqq \liminf _{n \rightarrow \infty} Q_{n}(\alpha) \leqq \limsup _{n \rightarrow \infty} Q_{n}(\alpha) \leqq \sigma(\alpha+\epsilon),
$$

provided $\alpha-\epsilon$ and $\alpha+\epsilon$ are continuity points of $\sigma(\alpha)$. Since $\epsilon$ can be made arbitrarily small we have

$$
\lim _{n \rightarrow \infty} Q_{n}(\alpha)=\sigma(\alpha)
$$

at each continuity point of $\sigma(\alpha)$.

Because of the complexity of the Laplace transform of $\sigma(\alpha)$ it does not appear to be quite easy to prove that $\sigma$ is continuous for all $\alpha$.

3. Reduction of the problem to solving an integral equation( $\left.{ }^{3}\right)$. Let $X_{1}, X_{2}, X_{3}, \ldots$ be independent random variables such that

(3) The procedure used in this section is almost identical with that used by the present author in his note Random walk in the presence of absorbing barriers, Annals of Mathematical Statistics vol. 16 (1945) pp. 62-67. This procedure has been used a great deal in statistical mechanics; see the reference to E. W. Montroll in the note just cited. 


$$
\text { Prob. }\left\{X_{j}<\alpha\right\}=2^{-1 / 2} \int_{-\infty}^{\alpha} e^{-2^{1 / 2}|u|} d u \quad(j=1,2, \cdots) .
$$

We can easily see that

$$
\bar{X}_{j}=0, \quad \overline{X_{j}^{2}}=1 .
$$

Thus in view of considerations of $\$ 2$ we have

$\lim _{x \rightarrow \infty} E\left(\exp \left\{-\frac{z}{n^{z / 2}}\left(\left|X_{1}\right|+\left|X_{1}+X_{2}\right|+\cdots+\left|X_{1}+\cdots+X_{n}\right|\right)\right\}\right)$
$=\int_{0}^{\infty} e^{-z \alpha} d \sigma(\alpha) \quad(z>0)$.

The reason for selecting the particular distribution (13) is that we are able to calculate explicitly the average

$A_{n}(z)=E\left(\exp \left\{-\frac{z}{n^{3 / 2}}\left(\left|X_{1}\right|+\left|X_{1}+X_{2}\right|+\cdots+\left|X_{1}+\cdots+X_{n}\right|\right)\right\}\right)$.

We have

$$
\begin{aligned}
A_{n}(z)=2^{-n / 2} \int_{-\infty}^{\infty} \cdots \int_{-\infty}^{\infty} \exp \left\{-\frac{z}{n^{3 / 2}}\left(\left|x_{1}\right|+\cdots+\left|x_{1}+\cdots+x_{n}\right|\right)\right\} \\
\quad \exp \left\{-2^{1 / 2}\left(\left|x_{1}\right|+\left|x_{2}\right|+\cdots+\left|x_{n}\right|\right)\right\} d x_{1} \cdots d x_{n} .
\end{aligned}
$$

Making the change of variables

$$
y_{1}=2^{1 / 2} x_{1}, y_{2}=2^{1 / 2}\left(x_{1}+x_{2}\right), \cdots, y_{n}=2^{1 / 2}\left(x_{1}+\cdots+x_{n}\right)
$$

we see that

$$
\begin{aligned}
A_{n}(z) & =\frac{1}{2^{n}} \int_{-\infty}^{\infty} \cdots \int_{-\infty}^{\infty} \exp \left\{-\frac{z}{2^{1 / 2} n^{3 / 2}}\left(\left|y_{1}\right|+\left|y_{2}\right|+\cdots+\left|y_{n}\right|\right)\right. \\
& \cdot \exp \left\{-\left(\left|y_{1}\right|+\left|y_{2}-y_{1}\right|+\cdots+\left|y_{n}-y_{n-1}\right|\right)\right\} d y_{1} \cdots d y_{n} .
\end{aligned}
$$

Let $\beta=z /(2 n)^{8 / 2}$ and

$$
K(s, t)=e^{-\beta|s|} e^{-|\sigma-t|} e^{-\beta|t|} / 2 .
$$

We see that the integrand of (14) is equal to

$$
K\left(0, y_{1}\right) K\left(y_{1}, y_{2}\right) \cdots K\left(y_{n-1}, y_{n}\right) e^{-\beta\left|y_{n}\right|} .
$$

Denoting by $\lambda_{1}, \lambda_{2}, \lambda_{3}, \cdots$ the eigenvalues and by $f_{1}(s), f_{2}(s), f_{3}(s), \cdots$ the normalized eigenfunctions of the integral equations

$$
\frac{1}{2} \int_{-\infty}^{\infty} e^{-\beta|\delta|} e^{-|\delta-t|} e^{-\beta|t|} f(t) d t=\lambda f(s),
$$

we find easily that 


$$
A_{n}(z)=\sum_{j=1}^{\infty} \lambda_{j}^{n} f_{j}(0) \int_{-\infty}^{\infty} f_{j}(t) e^{-\beta|t|} d t, \quad \beta=\frac{z}{(2 n)^{3 / 2}} .
$$

The problem is now reduced to finding eigenvalues and eigenfunctions of the integral equation (15).

4. Solution of the integral equation. In a recent paper $\left({ }^{4}\right) \mathrm{Mr}$. M. L. Juncosa discusses an analogous integral equation

$$
\int_{0}^{\infty} e^{-\beta s} e^{-|\sigma-t|} e^{-\beta t} f(t) d t=\lambda f(s)
$$

which I have encountered in some researches on random noise in radio receivers $\left({ }^{5}\right)$. His calculations are applicable here with only minor modifications, and it seems therefore sufficient to just state the result.

Let $r_{1}, r_{2}, r_{3}, \cdots$ be the positive roots of $J_{1 / \beta}(x)=0$ and $t_{1}, t_{2}, t_{3}, \cdots$ the positive roots of

$$
J_{1 / \beta}^{\prime}(x)=0 ;
$$

then the eigenvalues of (15) are

$$
1 /\left(r_{1} \beta\right)^{2}, 1 /\left(t_{1} \beta\right)^{2}, 1 /\left(r_{2} \beta\right)^{2}, 1 /\left(t_{2} \beta\right)^{2}, \cdots
$$

and the corresponding eigenfunctions are

$$
\frac{\operatorname{sign} s J_{1 / \beta}\left(r_{1} e^{-\beta|\bullet|}\right) e^{-\beta|\bullet|}}{M_{1}}, \frac{J_{1 / \beta}\left(t_{1} e^{-\beta|\varepsilon|}\right) e^{-\beta|\&|}}{N_{1}}, \ldots
$$

where $M_{1}, N_{1}, \ldots$ are normalizing factors.

Noticing that the eigenfunctions corresponding to $r_{1}, r_{2}, \cdots$ are 0 for $s=0$, we see that (16) can be written in the form

$$
A_{n}(z)=\sum_{j=1}^{\infty} \frac{1}{\left(\beta t_{j}\right)^{2 n}} \frac{J_{1 / \beta}\left(t_{j}\right) \int_{-\infty}^{\infty} J_{1 / \beta}\left(t_{j} e^{-\beta|\varepsilon|}\right) e^{-2 \beta|\varepsilon|} d s}{N_{j}^{2}} .
$$

We have

$$
\begin{gathered}
\int_{-\infty}^{\infty} J_{1 / \beta}\left(t_{j} e^{-\beta|\&|}\right) e^{-2 \beta|\&|} d s=\frac{2}{\beta} \int_{0}^{1} x J_{1 / \beta}\left(t_{j} x\right) d x \\
N_{j}^{2}=\int_{-\infty}^{\infty} J_{1 / \beta}^{2}\left(t_{j} e^{-\beta|\&|}\right) e^{-2 \beta|\&|} d s=\frac{2}{\beta} \int_{0}^{1} x J_{1 / \beta}^{2}\left(t_{j} x\right) d x=\frac{1}{\beta}\left(1-\frac{1}{\beta^{2} t_{j}^{2}}\right) J_{1 / \beta}^{2}\left(t_{j}\right)
\end{gathered}
$$

(4) Duke Math. J. vol. 12 (1945) pp. 465-471.

(5) A paper on this subject is being prepared for publication in the Physical Reviews by Dr. A. J. F. Siegert and the present author. 
and hence

$$
A_{n}(z)=2 \sum_{j=1}^{\infty} \frac{1}{\left(\beta t_{j}\right)^{2 n}} \frac{\int_{0}^{1} x J_{1 / \beta}\left(t_{j} x\right) d x}{\left(1-\frac{1}{\beta^{2} t_{j}^{2}}\right) J_{1 / \beta}\left(t_{j}\right)}, \beta=\frac{z}{(2 n)^{8 / 2}} .
$$

5. Auxiliary facts about Bessel functions. In order to find the limit of $A_{n}(z)$, as $n \rightarrow \infty$, we need various formulas and estimates involving Bessel functions. Some of the results appear to be new and may be of independent interest.

In this section constant references are made to the old edition of Watson's standard treatise Theory of Bessel functions and these references are made in text by giving in parentheses the page and sometimes the number of the formula.

I. For $y \geqq 0$ we have

$$
\lim _{n \rightarrow \infty} \nu^{1 / 3} J_{\nu}\left(\nu+y \nu^{1 / 3}\right)=\frac{(2 y)^{1 / 2}}{3}\left\{J_{-1 / 3}\left(\frac{(2 y)^{3 / 2}}{3}\right)+J_{1 / 3}\left(\frac{(2 y)^{3 / 2}}{3}\right)\right\},
$$

the convergence being uniform in every finite $y$-interval. This fact follows almost immediately from Watson's formula (252 (5)) which states that for $0 \leqq \xi<\pi / 4$ one has

$$
\begin{aligned}
& J_{\nu}(\nu \sec \xi)=\frac{\tan \xi}{3} \cos \left\{\nu\left(\tan \xi-\frac{\tan ^{8} \xi}{3}-\xi\right)\right\} \\
& \cdot\left[J_{-1 / 3}\left(\frac{\nu \tan ^{3} \xi}{3}\right)+J_{1 / 3}\left(\frac{\nu \tan ^{8} \xi}{3}\right)\right] \\
&+ 3^{-1 / 2} \tan \xi \sin \left\{\nu\left(\tan \xi-\frac{\tan ^{3} \xi}{3}-\xi\right)\right\} \\
& \cdot\left[J_{-1 / 3}\left(\frac{\nu \tan ^{3} \xi}{3}\right)-J_{1 / 3}\left(\frac{\nu \tan ^{8} \xi}{3}\right)\right]+\frac{24 \theta}{\nu}
\end{aligned}
$$

where

$$
|\theta| \leqq 1
$$

In fact, putting

$$
\sec \xi=1+y / \nu^{2 / 3}
$$

we have

$$
\tan \xi=\left(\sec ^{2} \xi-1\right)^{1 / 2} \sim \frac{(2 y)^{1 / 2}}{\nu^{1 / 3}}
$$

and our assertion follows from the elementary facts that

$$
\nu^{1 / 8} \tan \xi \rightarrow(2 y)^{1 / 2}
$$


and

$$
\nu\left(\tan \xi-\frac{\tan ^{3} \xi}{3}-\xi\right) \rightarrow 0,
$$

and that both limits are approached uniformly in finite $y$-intervals.

II. Let $\gamma_{1}, \gamma_{2}, \gamma_{3}, \ldots$ be the positive zeros of

$$
P(y)=\frac{(2 y)^{1 / 2}}{3}\left\{J_{-1 / 3}\left(\frac{(2 y)^{3 / 2}}{3}\right)+J_{1 / 3}\left(\frac{(2 y)^{3 / 2}}{3}\right)\right\}
$$

and $\delta_{1}, \delta_{2}, \ldots$ the positive zeros of the derivative $P^{\prime}(y)$; then

$$
r_{j}=\nu+\gamma_{j} \nu^{1 / 3}+o\left(\nu^{1 / 3}\right), \quad t_{j}=\nu+\delta_{j} \nu^{1 / 3}+o\left(\nu^{1 / 3}\right) .
$$

Considerably more seems to be known. Watson, for instance, shows that $O\left(\nu^{1 / 8}\right)$ can be replaced by $O\left(\nu^{-1 / 3}\right)$ but he carries out the proof only for $j=1$. Jahnke and Emde cite asymptotic formulas for $r_{1}, r_{2}, r_{3}$ and $t_{1}$.

For the sake of completeness we give the proof of (20) especially since it follows rather easily from (18).

Let $\epsilon>0$ be such that $P\left(\gamma_{1}-\epsilon\right) P\left(\gamma_{1}+\epsilon\right)<0$ and $P\left(\gamma_{2}-\epsilon\right) P\left(\gamma_{2}+\epsilon\right)<0$. If we define $P(0)$ as the limit of $P(y)$ as $y \rightarrow 0$ (this limit can be easily seen to exist) and denote by $\mu(\epsilon)$ the greatest lower bound of $|P(y)|$ in the set consisting of the two closed intervals $\left(0, \gamma_{1}-\epsilon\right),\left(\gamma_{1}+\epsilon, \gamma_{2}-\epsilon\right)$, then choosing $\nu$ large enough so that

$$
\begin{aligned}
\left|\nu^{1 / 3} J_{\nu}\left(\nu+y \nu^{1 / 3}\right)-P(y)\right| & <\min \left(\mu(\epsilon) / 2,\left|P\left(\gamma_{1}-\epsilon\right)\right| / 2,\right. \\
& \left.\left|P\left(\gamma_{1}+\epsilon\right)\right| / 2,\left|P\left(\gamma_{2}-\epsilon\right)\right| / 2,\left|P\left(\gamma_{2}+\epsilon\right)\right| / 2\right)
\end{aligned}
$$

for $0 \leqq y \leqq \gamma_{2}+\epsilon$, we see that a root of $J_{\nu}\left(\nu+y \nu^{1 / 8}\right)$ must lie in $\left(\gamma_{1}-\epsilon, \gamma_{1}+\epsilon\right)$, another root in $\left(\gamma_{2}-\epsilon, \gamma_{2}+\epsilon\right)$, and no root falls within $\left(0, \gamma_{1}-\epsilon\right)$ and $\left(\gamma_{1}+\epsilon\right.$, $\left.\gamma_{2}-\epsilon\right)$. If more than one root would lie in either $\left(\gamma_{1}-\epsilon, \gamma_{1}+\epsilon\right)$ or $\left(\gamma_{2}-\epsilon\right.$, $\left.\boldsymbol{\gamma}_{2}+\epsilon\right)$ we should get a contradiction with the well known fact that differences between consecutive roots of $J_{\nu}(x)$ for $\nu>1 / 2$ form a decreasing sequence. It follows that $\gamma_{1}$ falls within $\left(\gamma_{1}-\epsilon, \gamma_{1}+\epsilon\right)$ and $\gamma_{2}$ within $\left(\gamma_{2}-\epsilon, \gamma_{2}+\epsilon\right)$, provided $\nu$ is sufficiently large. This proves the first part of (20) for $j=1,2$ and it is obvious that the reasoning can be extended to all $j$.

To prove the corresponding statement for the $t_{j}$ 's we first notice that

$$
\nu<t_{1}<r_{1}<t_{2}<r_{2}<\cdots
$$

and

$$
0<\delta_{1}<\gamma_{1}<\delta_{2}<\gamma_{2}<\cdots .
$$

Let $\epsilon>0$ be small enough and such that

$$
\left|P\left(\delta_{j}-\epsilon\right)\right|<\left|P\left(\delta_{j}\right)\right| \text { and }\left|P\left(\delta_{j}+\epsilon\right)\right|<\left|P\left(\delta_{j}\right)\right| \text {. }
$$

Then for sufficiently large $\nu$ 


$$
\nu^{1 / 3}\left|J_{\nu}\left(\nu+\left(\delta_{j}-\epsilon\right) \nu^{1 / 3}\right)\right|<\nu^{1 / 3}\left|J_{\nu}\left(\nu+\delta_{j} \nu^{1 / 3}\right)\right|
$$

and

$$
\nu^{1 / 3}\left|J_{\nu}\left(\nu+\left(\delta_{j}+\epsilon\right) \nu^{1 / 3}\right)\right|<\nu^{1 / 3}\left|J_{\nu}\left(\nu+\delta_{j} \nu^{1 / 3}\right)\right| .
$$

Thus a turning point of $J_{\nu}(x)$ falls within the interval $\left(\nu+\left(\delta_{j}-\epsilon\right) \nu^{1 / 3}\right.$, $\left.\nu+\left(\delta_{j}+\epsilon\right) \nu^{1 / 3}\right)$, provided $\nu$ is sufficiently large. Now, $\nu$ can be made, if necessary, larger so that also

$$
r_{j-1}<\nu+\left(\delta_{j}-\epsilon\right) \nu^{1 / 3}<\nu+\left(\delta_{j}+\epsilon\right) \nu^{1 / 3}<r_{j}
$$

and since only one turning point of $J_{j}(x)$ lies between $r_{j-1}$ and $r_{j}$ we see that

$$
\nu+\left(\delta_{j}-\epsilon\right) \nu^{1 / 3}<t_{j}<\nu+\left(\delta_{j}+\epsilon\right) \nu^{1 / 3} .
$$

This completes the proof of (20).

III. We have

$$
\lim _{\nu \rightarrow \infty} \frac{1}{\nu} \int_{\nu}^{t_{i}} x J_{\nu}(x) d x=\int_{0}^{\delta_{i}} P(y) d y .
$$

Let $x=\nu+y \nu^{1 / 8}$ and $y_{i}=\left(t_{i}-\nu\right) / \nu^{1 / 3}$; then

$$
\frac{1}{\nu} \int_{\nu}^{t_{j}} x J_{\nu}(x) d x=\int_{0}^{y_{i}}\left(1+\frac{y}{\nu^{2 / 3}}\right) \nu^{1 / 3} J_{\nu}\left(\nu+y \nu^{1 / 3}\right) d y .
$$

From (20) it follows that $y_{j} \rightarrow \delta_{j}$ as $\nu \rightarrow \infty$ and since $\nu^{1 / 3} J_{\nu}\left(\nu+y \nu^{1 / 3}\right)$ approaches $P(y)$ uniformly in every finite interval, (21) follows.

IV. We have

$$
\begin{aligned}
\lim _{\nu \rightarrow \infty} \frac{1}{\nu} \int_{0}^{\nu} x J_{\nu}(x) d x & =\frac{1}{3^{1 / 2} \pi} \int_{0}^{\infty} y^{2} K_{1 / 3}\left(\frac{y^{2}}{3}\right) d y \\
& =\frac{1}{3^{1 / 2} \pi} \int_{0}^{\infty} K_{1 / 3}(y) d y=\frac{1}{3} .
\end{aligned}
$$

We use Watson's formula (250 (4))

$$
\begin{gathered}
J_{\nu}(\nu \operatorname{sech} \alpha)=\frac{\tanh \alpha}{3^{1 / 2} \pi} \exp \left\{\nu\left(\tanh \alpha+\frac{1}{3} \tanh ^{3} \alpha-\alpha\right)\right\} K_{1 / 3}\left(\frac{\nu}{3} \tanh ^{8} \alpha\right) \\
+\frac{3 \theta_{1}}{\nu} \exp \{\nu(\tanh \alpha-\alpha)\},
\end{gathered}
$$

where $\left|\theta_{1}\right| \leqq 1$. Since this formula is valid for all $\alpha>0$ we can put

$$
\operatorname{sech} \alpha=x / \nu
$$$$
(0 \leqq x \leqq \nu)
$$

obtaining

$$
\tanh \alpha=\left(1-x^{2} / \nu^{2}\right)^{1 / 2}
$$


If we use these expressions in the integral

$$
\int_{0}^{1} x J_{\nu}(x) d x
$$

and then make the substitution

$$
y=\nu^{1 / 3}\left(1-x^{2} / \nu^{2}\right)^{1 / 2}
$$

we obtain in a perfectly elementary fashion

$$
\begin{aligned}
& \frac{1}{\nu} \int_{0}^{\nu} x J_{\nu}(x) d x \\
& \quad=\frac{1}{3^{1 / 2} \pi} \int_{0}^{\nu 1 / 3} y^{2} \exp \left\{\nu\left(\frac{y}{\nu^{1 / 8}}+\frac{1}{3} \frac{y^{3}}{\nu}-\tanh ^{-1} y\right)\right\} K_{1 / 8}\left(\frac{y^{2}}{3}\right) d y_{-}^{-}+{ }_{L} R_{\nu}
\end{aligned}
$$

where

$$
\left|R_{\nu}\right|<\frac{3}{\nu^{2 / 3}} \int_{0}^{\nu 1 / 8} y \exp \left\{\nu\left(\frac{y}{\nu^{1 / 3}}-\tanh ^{-1} \frac{y}{\nu^{1 / 3}}\right)\right\} d y .
$$

It is quite easy to see that $R_{p} \rightarrow 0$. Since $y^{2} K_{1 / 3}\left(y^{8} / 3\right)$ is positive and integrable in $(0, \infty)$ and since for each $y$

$$
\exp \left\{\nu\left(\frac{y}{\nu^{1 / 3}}+\frac{1}{3} \frac{y^{3}}{\nu}-\tanh ^{-1} \frac{y}{\nu^{1 / 8}}\right)\right\} \leqq 1
$$

and

$$
\lim _{\nu \rightarrow \infty} \exp \left\{\nu\left(\frac{y}{\nu^{1 / 3}}+\frac{1}{3} \frac{y^{3}}{\nu}-\tanh ^{-1} \frac{y}{\nu^{1 / 3}}\right)\right\}=1,
$$

we see that the first part of (22) follows.

It remains to prove that

$$
\frac{1}{3^{1 / 2} \pi} \int_{0}^{\infty} K_{1 / 3}(y) d y=\frac{1}{3} .
$$

This, however, follows in a few steps from (388(8)).

V. Combining the results of III and IV we get

$$
\lim _{\nu \rightarrow \infty} \frac{1}{\nu} \int_{0}^{t_{j}} x J_{\nu}(x) d x=\frac{1}{3}\left(1+3 \int_{0}^{\delta_{j}} P(y) d y\right) .
$$

In the same way it can be shown that

$$
\lim _{\nu \rightarrow \infty} \frac{1}{\nu} \int_{0}^{r_{i}} x J_{\nu}(x) d x=\frac{1}{3}\left(1+3 \int_{0}^{\gamma_{j}} P(y) d y\right) .
$$

VI. We now need the fact that for $x>0$ and $\nu>0$ the consecutive areas 
bounded by the $x$-axis and the graph of $J_{\nu}(x) / x$ form a decreasing sequence. This can be easily proved using Sturm's method; it is also contained in a more general theorem of R. G. Cooke $\left({ }^{6}\right)$.

As an immediate consequence we obtain

$$
0 \leqq \int_{0}^{t} \frac{J_{\nu}(x)}{x} d x \leqq \int_{0}^{n_{1}} \frac{J_{\nu}(x)}{x} d x .
$$

By a reasoning entirely analogous to the one used in III and IV we get

$$
\lim _{n \rightarrow \infty} \nu \int_{0}^{n} \frac{J_{\nu}(x)}{x} d x=\frac{1}{3}\left(1+3 \int_{0}^{\gamma_{1}} P(y) d y\right) .
$$

Thus an absolute constant $A$ exists such that

$$
\nu \int_{0}^{n} \frac{J_{\nu}(x)}{x} d x<A,
$$

and therefore in view of (25) we have for all $t$ and $\nu \geqq 1$.

$$
0 \leqq \int_{0}^{t} \frac{J_{\nu}(x)}{x} d x<\frac{A}{\nu} \text {. }
$$

VII. For all $j$ and $\nu \geqq 1$ we have

$$
0 \leqq \int_{0}^{t_{j}} x J_{\nu}(x) d x<A \nu .
$$

In fact, writing the Bessel differential equation in the form

$$
\frac{d}{d x}\left(x J_{r}^{\prime}(x)\right)+\left(x-\frac{\nu^{2}}{x}\right) J_{\nu}(x)=0
$$

and integrating between 0 and $t_{i}$ we obtain

$$
\int_{0}^{t_{j}} x J_{\nu}(x) d x=\nu^{2} \int_{0}^{t_{j}} \frac{J_{\nu}(x)}{x} d x .
$$

This together with (26) implies (27).

VIII. We have

$$
\lim _{x \rightarrow \infty} \frac{\int_{0}^{1} x J_{\nu}\left(t_{j} x\right) d x}{\left(1-\nu^{2} / t_{j}^{2}\right) J_{\nu}\left(t_{j}\right)}=\frac{1}{2} \frac{1+3 \int_{0}^{\delta_{j}} P(y) d y}{3 \delta_{j} P\left(\delta_{j}\right)}
$$

and for sufficiently large $\nu$

(8) Gibbs' phenomenon in Fourier-Bessel series and integrals, Proc. London Math. Soc. vol. 27 (1928) pp. 171-192, in particular the theorem on p. 178. 


$$
\left|\frac{\int_{0}^{1} x J_{\nu}\left(t_{j} x\right) d x}{\left(1-\nu^{2} / t^{2}\right) J_{\nu}\left(t_{j}\right)}\right|<C,
$$

where $C$ is an absolute constant.

We write

$$
\frac{\int_{0}^{1} x J_{\nu}\left(t_{j} x\right) d x}{\left(1-\nu^{2} / t_{j}^{2}\right) J_{\nu}\left(t_{j}\right)}=\frac{\frac{1}{\nu} \int_{0}^{t_{j}} x J_{\nu}(x) d x}{\left(t_{j}^{2}-\nu\right) J_{\nu}\left(t_{j}\right) / \nu} .
$$

By (23) the numerator approaches $1 / 3$ of the numerator of the right-hand side of (28). Since $t_{j}=\nu+\delta_{j} \nu^{1 / 2}+o\left(\nu^{1 / 3}\right)$ and $\left(t_{j}+\nu\right) / \nu \sim 2$ we deduce from (18) that

Thus (28) follows.

$$
\lim _{x \rightarrow \infty} \frac{1}{\nu} J_{i}\left(t_{j}\right)\left(t_{j}^{2}-\nu^{2}\right)=2 \delta_{j} P\left(\delta_{j}\right) \text {. }
$$

To prove (29) we refer to the known fact (488) that for a fixed $\nu>0$ the numbers

$$
\left(t_{j}^{2}-\nu^{2}\right)^{1 / 4}\left|J_{\nu}\left(t_{j}\right)\right|
$$

form an increasing sequence. Thus

$$
\left(t_{j}^{2}-\nu^{2}\right)^{1 / 4}\left|J_{\nu}\left(t_{j}\right)\right| \geqq\left(t_{1}^{2}-\nu^{2}\right)^{1 / 4}\left|J_{\nu}\left(t_{1}\right)\right|
$$

and hence

$$
\left(t_{j}^{2}-\nu^{2}\right)\left|J_{\nu}\left(t_{j}\right)\right| / \nu \geqq\left(t_{1}^{2}-\nu^{2}\right)\left|J_{\nu}\left(t_{1}\right)\right| / \nu
$$

for all $j$ and $\nu>0$.

As noticed above

$$
\lim _{\nu \rightarrow \infty} \frac{1}{\nu}\left(t_{1}^{2}-\nu^{2}\right)\left|J_{\nu}\left(t_{1}\right)\right|=2 \delta_{1}\left|P\left(\delta_{1}\right)\right|>0
$$

and hence a positive absolute constant $B$ can be chosen such that for suffciently large $\nu$

$$
\left(t_{1}^{2}-\nu^{2}\right)\left|J_{\nu}\left(t_{1}\right)\right| / \nu>B>0 .
$$

Combining this with (27) we obtain (29) with $C=A / B$.

IX. If

$$
\nu=1 / \beta=(2 n)^{3 / 2} / z
$$

we have

$$
\lim _{n \rightarrow \infty}\left(\beta t_{j}\right)^{2 n}=\exp \left\{\delta_{j} z^{2 / 8}\right\}
$$

In fact 


$$
\beta t_{j}=1+\delta_{j} \nu^{-2 / 3}+o\left(\nu^{-2 / 3}\right)=1+\delta_{j} z^{2 / 3} / 2 n+o(1 / n)
$$

and (30) follows.

$\mathrm{X}$. We finally need the following estimate

$$
r_{j}^{2}>\nu^{2}+(2 \pi j)^{2 / 3} \nu^{4 / 3}
$$

for all $j$ and $\nu>0$. This result appears to be new and of independent interest. We prove it using Sturm's method.

Let $r_{i}$ and $r_{j+1}$ be two consecutive positive roots of $J_{v}(x)$ and let

$$
\theta_{j}=\log \frac{r_{j}}{\nu}, \quad \theta_{j+1}=\log \frac{r_{j+1}}{\nu} .
$$

Obviously $\theta_{j}$ and $\theta_{j+1}$ are consecutive roots of $u(\theta)=J_{\nu}\left(\nu e^{\theta}\right)$. It is well known that

$$
u^{\prime \prime}(\theta)+\nu^{2}\left(e^{2 \theta}-1\right) u(\theta)=0 .
$$

For $\theta_{j}<\theta<\theta_{j+1}$ we have

$$
\nu^{2}\left(e^{2 \theta}-1\right)<\nu^{2}\left(e^{2 \theta j+1}-1\right)=r_{j+1}^{2}-\nu^{2} .
$$

Let $v(\theta)=\sin \left(r_{j+1}^{2}-\nu^{2}\right)^{1 / 2}\left(\theta-\theta_{j}\right)$ and hence

$$
v^{\prime \prime}(\theta)+\left(r_{j+1}^{2}-\nu^{2}\right) v(\theta)=0 .
$$

We have $u\left(\theta_{j}\right)=v\left(\theta_{j}\right)=0$ and hence by Sturm's theorem the root of $v(\theta)$, which is next to $\theta_{j}$ must be smaller than $\theta_{j+1}$. Thus

or

$$
\theta_{j+1}>\theta_{j}+\frac{\pi}{\left(r_{j+1}^{2}-\nu^{2}\right)^{1 / 2}}
$$

$$
\log \frac{r_{j+1}}{r_{j}}>\frac{\pi}{\left(r_{j+1}^{2}-\nu^{2}\right)^{1 / 2}} \text {. }
$$

It now follows that

$$
\log \frac{r_{j}}{r_{1}}>\pi \sum_{k=2}^{j} \frac{1}{\left(r_{k}^{2}-\nu^{2}\right)^{1 / 2}}>\frac{\pi j}{\left(r_{j}^{2}-\nu^{2}\right)^{3 / 2}}
$$

and since $r_{1}>\nu$ we have

$$
\frac{r_{j}}{\nu}>\exp \left\{\frac{\pi j}{\left(r_{j}^{2}-\nu^{2}\right)^{1 / 2}}\right\} .
$$

Hence

$$
\frac{r_{j}^{2}}{\nu^{2}}>\exp \left\{\frac{2 \pi j}{\left(r_{j}^{2}-\nu^{2}\right)^{1 / 2}}\right\}>1+\frac{2 \pi j}{\left(r_{j}^{2}-\nu^{2}\right)^{1 / 2}}
$$

which leads immediately to 


$$
\left(r_{j}^{2}-\nu^{2}\right)^{3 / 2}>2 \pi j \nu^{2}
$$

and (31) follows.

Since $t_{j}>r_{j-1}(j>1)$ and $t_{1}>\nu$ we have

$$
t_{j}^{2}>\nu^{2}+(2 \pi(j-1))^{2 / 3} \nu^{4 / 3} \text {. }
$$

If we put

$$
\nu=\frac{1}{\beta}=\frac{(2 n)^{3 / 2}}{z}
$$

we obtain

$$
\beta^{2} t_{j}^{2}>1+\frac{(2 \pi z(j-1))^{2 / 3}}{2 n}
$$

and hence for $n \geqq 3$

$$
\left(\beta t_{j}\right)^{2 n}>\frac{n(n-1)(n-2)}{3 !} \frac{2 \pi z(j-1)^{2}}{8 n^{3}}>\frac{\pi z}{72}(j-1)^{2} .
$$

6. Passage to the limit. Recalling formula (17)

$$
A_{n}(z)=2 \sum_{j=1}^{\infty} \frac{1}{\left(\beta t_{j}\right)^{2 n}} \frac{\int_{0}^{1} x J_{1 / p}\left(t_{j} x\right) d x}{\left(1-1 / \beta^{2} t_{j}^{2}\right) J_{1 / \beta}\left(t_{j}\right)}, \quad \beta=\frac{z}{(2 n)^{z / 2}},
$$

we can now find easily the limit of $A_{n}(z)$, as $n \rightarrow \infty$. Using (28) and (30) we obtain formally

$$
\lim _{n \rightarrow \infty} A_{n}(z)=\sum_{j=1}^{\infty} \exp \left\{-\delta_{j} z^{2 / 8}\right\} \frac{1+3 \int_{0}^{\delta_{i}} P(y) d y}{3 \delta_{j} P\left(\delta_{j}\right)} .
$$

The justification of taking the limit term by term is provided by the estimates (29) and (32) inasmuch as these estimates clearly imply that for $n \geqq 3$ and $j \geqq 2$ the terms of series (17) are majorized by

$$
\frac{72 C}{\pi z} \frac{1}{(j-1)^{2}}
$$

Cornell University, ITHACA, N. Y. 